\title{
Arquitectura, urbanismo y obras públicas civiles y militares en el Atlas Geográfico de España de Tomás López de 1804
}

\author{
Architecture, urbanism and civil and military public works in the \\ Geographic Atlas of Spain (1804) of Tomas Lopez
}

$\underline{\text { C. San-Antonio-Gómez }}{ }^{(*)}$, J. S. Fernández-Sánchez ${ }^{(* *)}$, F. Manzano-Agugliaro ${ }^{(* *)}$

\section{RESUMEN}

El objetivo que planteamos es analizar cómo Tomás López representó en su Atlas Geográfico de España (AGE) de 1804, la arquitectura, el urbanismo y las obras públicas civiles y militares, un análisis hasta el momento inédito. Para ello se han caracterizado los diferentes símbolos cartográficos que aparecen en los mapas, que constituyen los parámetros de referencia del análisis y transmiten información fidedigna de la forma y significado de lo representado. Para esas tipologías se han encontrado ciento trece símbolos diferentes, lo que da idea de la minuciosidad y riqueza de la información contenida en los mapas. Dieciocho de ellos, son equivalentes con otros tantos símbolos cartográficos de Chevalier, maestro de D’Anville, de quien Tomás López fue discípulo en París. También hay una similitud entre cuatro símbolos del AGE con sus correspondientes signos de las Ordenanzas del Real Cuerpo de Ingenieros de 1803.

Palabras clave: Arquitectura; urbanismo; obras públicas civiles y militares; Tomás López; Atlas Geográfico de España; representación.

\section{ABSTRACT}

This manuscript presents a novel analysis that deals about how architecture, urban planning and civil and military public works are represented in Geographic Atlas of Spain 1804 (AGE) of Tomas Lopez. For this it has been characterized different map symbols appearing on maps, which are the benchmarks for analysis and transmit accurate information on the shape and meaning of what is represented. So, more than one hundred different symbols were found. This gives an idea of the great detail and richness of the information contained in AGE. A deeper analysis shows that eighteen of them are equivalent to other previous cartographic symbols. These were from Chevalier, D'Anville teacher, who in turn was the teacher Thomas Lopez in Paris. Additionally, we found that there are several symbols with similarity to their corresponding of the Ordinances of the Royal Corps of Engineers from 1803.

Keywords: Architecture; urbanism; civilian and military public works; Tomas Lopez; Geographic Atlas of Spain; representation.

(*) Universidad Politécnica de Madrid.

${ }^{(* *)}$ Junta de Andalucía.

${ }^{(* * *)}$ Universidad de Almería.

Persona de contacto/Corresponding author: c.sanantonio@upm.es (C. San-Antonio-Gómez)

Cómo citar este artículo/Citation: San-Antonio-Gómez, C., Fernández-Sánchez, J. S., Manzano-Agugliaro, F. (2016). Arquitectura, urbanismo y obras públicas civiles y militares en el Atlas Geográfico de España de Tomás López de 1804. Informes de la Construcción, 68(542): e147, doi: http://dx.doi.org/10.3989/ic.15.075.

Licencia / License: Salvo indicación contraria, todos los contenidos de la edición electrónica de Informes de la Construcción se distribuyen bajo una licencia de uso y distribución Creative Commons Attribution License (CC BY) Spain 3.o. 


\section{INTRODUCCIÓN}

El Atlas Geográfico de España (AGE), de Tomás López, fue la culminación de más de tres décadas de ingente trabajo que pone en evidencia su titánica labor (1). Es la colección de mapas de España peninsular -en el que se incluye Portugal-y de las Baleares más detallada hecha hasta ese momento. Sus mapas son de gran belleza pero de escasa precisión (2). La forman 102 hojas con una completa información geográfica de miles de enclaves de los 36 territorios que la componían. Tomás López compuso su Atlas con información de diversas fuentes (historias locales, descripciones geográficas, mapas, etc.) y con el Interrogatorio que le enviaban los párrocos de determinados lugares, que incluía un mapa de la zona sin el menor rigor científico. Ya en gabinete, organizaba ese complejo material con el que daba forma definitiva a sus mapas (3).

La primera edición del Atlas se publicó en Madrid en 1804, cuando Tomás López ya había fallecido por lo que fueron sus hijos y su viuda los encargados de la edición que, posteriormente, tuvo otras (4). En la Biblioteca Nacional se conservan dos ejemplares del Atlas con signaturas $\mathrm{GMg} / 300$ y GMG/1190. Tiene 102 folios que contienen 38 mapas grabados en cobre. Sus escalas oscilan entre 1:100.000 y 1:650.000. Del $A G E$ se han publicado dos ediciones facsímile, la primera a cargo de Sanz Hermida (5) y la segunda de Hernando (6).

El $A G E$ era conocido en la Guerra de la Independencia, lo tenían para sus operaciones los ejércitos de Wellington (7) y de Napoleón, que lo utilizó como referencia para formar su propio Mapa de España y Portugal, a escala 1:500.000 (8). Ambos ejércitos comprobaron la inexactitud de sus mapas, algo que se convirtió en un lugar común entre los estudiosos. La magnitud de esos errores se ha cuantificado en $6,5 \mathrm{~km}$ de media para toda la obra, aumentando estos errores en las zonas interiores como la hoja del Reino de Jaén con 7,5 km (9) y más aún en las zonas alejadas de los caminos de postas, tal es el caso del mapa del Reino de Extremadura con 10 km (10).

Junto a esos errores, el $A G E$ adolecía de dos defectos: la disparidad de escalas y de meridianos de referencia, porque adoptó, indistintamente, el del Teide, la Isla del Hierro y el de Madrid. Para juzgar la producción cartográfica de Tomás López hay que situarse en su época y en sus circunstancias, y así se podrá concluir que si Tomás López no fue un geógrafo de primer orden, por lo menos ha prestado a la ciencia incontrastables servicios (11).

La bibliografía sobre Tomás López es abundantísima e incide, fundamentalmente, en aspectos biográficos e histórico-geográficos, generalmente referidos al contexto político-social del siglo xviII (12) y (13), pero no aborda el estudio de los recursos gráficos de sus mapas, que es el objetivo que nos proponemos. En los mapas históricos suelen representarse bellamente la topografía, la vegetación, las cartelas, escalas, etc., como en muchos mapas de la América hispana, que muestran una excelente cartografía pero sin el esfuerzo normativo realizado por Tomás López (14). Para ello, habría que clasificar los recursos gráficos más comunes en la historia de la cartografía para representar el relieve del terreno, la arquitectura, la ciudad, las obras públicas civiles y militares, la vegetación y ciertos dispositivos gráficos como cartelas, rosas de los vientos, escalas, colores, etc. (15).

Para examinar los recursos gráficos del $A G E$, ha sido necesario definir una metodología de análisis que consiste en la ca- racterización de los modelos tipológicos representados, como son: el relieve del terreno, las construcciones, la hidrología, la vegetación, etc. Esos modelos facilitan el análisis gráfico de la cartografía histórica y constituyen los parámetros de referencia con los que confrontar cualquier plano o mapa histórico. Las convenciones gráficas del AGE, transmiten información fidedigna tanto del significado de lo representado como de su forma, sin olvidar la precisión y la fiabilidad de esa información.

\section{MODELOS TIPOLÓGICOS Y SÍMBOLOS GRÁFICOS DEL AGE. IDENTIFICACIÓN Y REPRESENTACIÓN}

Para identificar cómo se representan en cualquier mapa histórico los diferentes símbolos o recursos gráficos que un cartógrafo utiliza para trasladar al documento, de manera irrefutable y no contradictoria, la realidad que quiere representar, es necesario definir una serie de modelos tipológicos que agrupen los convencionalismos más frecuentes que aparecen en los mapas. Esos recursos gráficos suelen ser comunes a todos los mapas históricos y permiten el análisis de ese rico legado patrimonial. Para el $A G E$ hemos definido los diez modelos tipológicos más repetidos: Geográficos, Topográficos, Cultivos y vegetación, Arquitectónicos, Urbanos, Obras ingeniería civil y Obras ingeniería militar, Administración religiosa, Administración civil y militar y Recursos naturales y producción industrial. Como veremos más adelante, en este artículo nos limitaremos a describir los contenidos gráficos de solamente cuatro de esos diez modelos: Arquitectónicos, Urbanos, Obras ingeniería civil y Obras ingeniería militar.

En cuanto a los símbolos cartográficos del $A G E$, aparecen en cada uno de sus mapas junto con las escalas gráficas, meridiano de referencia y el elenco de fuentes consultadas: mapas, respuestas a interrogatorios, historias locales, descripciones geográficas, personas que le facilitaron información, etc. Generalmente esos símbolos, que Tomás López llama señales, con algunas variaciones, son los mismos para cada mapa (Figuras 1 y 2). Las señales parecen basadas en la tabla de signos cartográficos convencionales de Chevalier, cartógrafo de Bossuet y profesor en el Collège de France, que propuso en 1707 a la Académie de Sciences de París, un método de levantamiento que retomaría el conocido cartógrafo francés D’Anville -con quien se formó Tomás López en sus nueve años de estancia en esa ciudad- que se denominó de la Topografía Eclesiástica (16). El método lo publicó la Académie con el título: Sur la Maniere de lever la carte d'un pays y consistía en que cada párroco levantara un mapa de su pueblo y alrededores. Para ello, Chevalier diseñó una plantilla de cartón que enviaba a los párrocos y que denominó «châssis orienté» (17), en la que figuraban los símbolos cartográficos (Figura 3) con objeto de homogeneizar la representación gráfica de los mapas (18).

En la Figura 4, vemos la equivalencia entre los símbolos cartográficos de Chevalier y de Tomás López, con la coincidencia de las simbologías con las que representan un mismo elemento gráfico. Se puede concluir que existe una notable coincidencia entre la representación gráfica de López con la de Chevalier.

Además de la semejanza entre los símbolos de Chevalier y Tomás López, encontramos también un paralelismo entre las 


\section{Explicacion de las señales.}

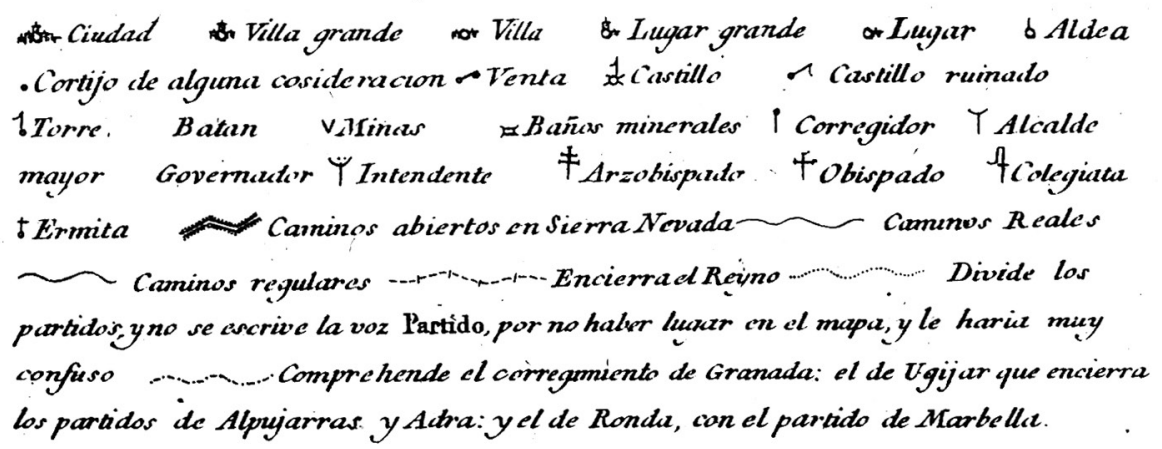

Figura 1. Símbolos (señales) del Reino de Granada (Lámina 68 del AGE).

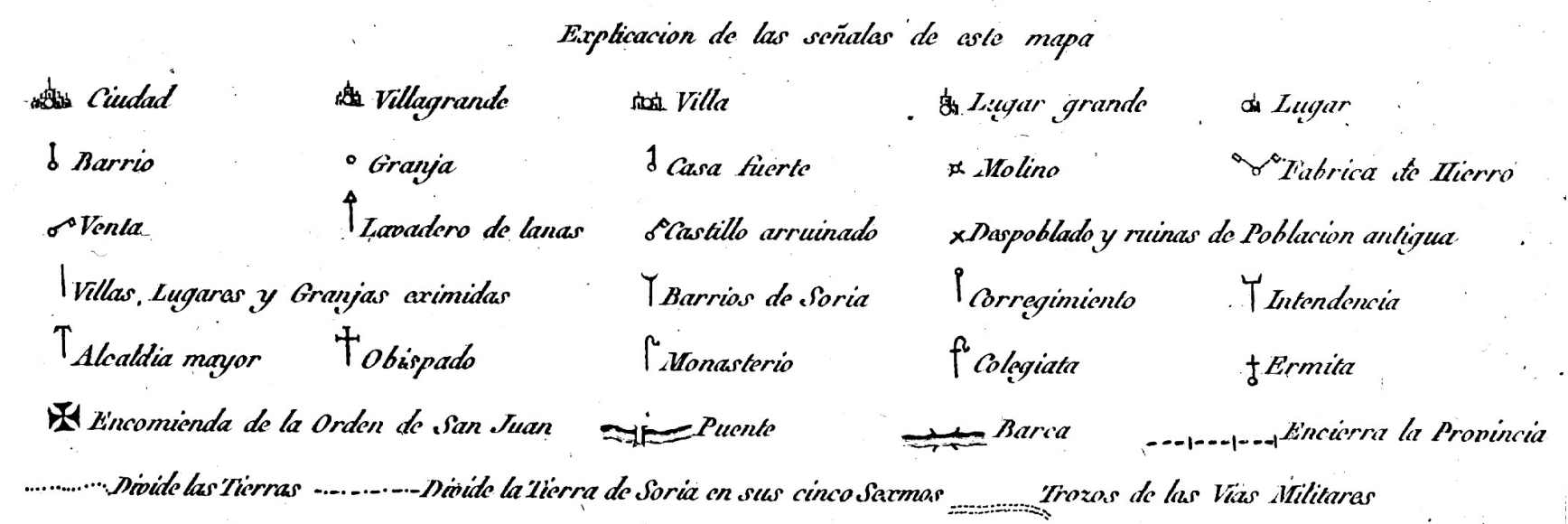

Figura 2. Símbolos (señales) de la Provincia de Soria (Lámina 18 del AGE).

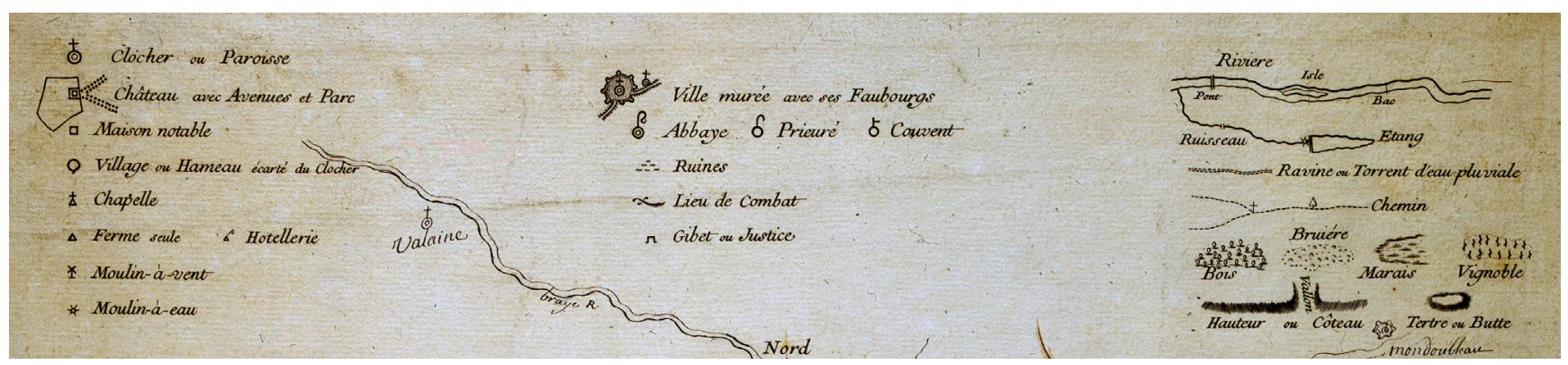

Figura 3. Signos cartográficos para la plantilla de Chevalier (Alinhac, 1973), extracto de Tracés manuscrits des environs de Savigny, Collection d'Anville, Bibliothèque Nationale de France.

señales del $A G E$ con algunos de los signos convencionales de las Ordenanzas del Real Cuerpo de Ingenieros del Ejército que Carlos IV aprobó en 1803 (19) (Figura 5), un año antes de la publicación del Atlas, que supusieron un notable avance en la reglamentación de formatos y signos convencionales respecto a los setenta y cinco señales de las Ordenanzas de 1768 (20). La analogía entre las dos simbologías puede verse en la Figura 6.

Los símbolos de los mapas del $A G E$, representan una serie de contenidos que, como anteriormente quedó dicho, agrupamos en diez modelos tipológicos. En este artículo nos limitaremos a describir los contenidos gráficos de cuatro de esos diez modelos tipológicos: Arquitectónicos, Urbanos, Obras ingeniería civil y Obras ingeniería militar. A continuación pasamos a describir cada uno de ellos.

\subsection{Modelo tipológico elementos arquitectónicos}

Para éste modelo se han caracterizado veintitrés elementos diferentes que reflejan tanto las construcciones de uso civil como las religiosas: Abadía, Baños, Capilla, Castillos, Castillos en ruinas, Castillos mayores, Castillo Moderno, Castillo antiguo, Colegiata, Convento, Convento de orden militar, Ermitas, Hospital, Iglesia colegiata, Molinos, Monasterio, Palacios, Plazas, Santuario, Templo, Torre o atalaya, Universidad, Venta. Cada uno de ellos se representa gráficamente en los mapas del $A G E$ con un símbolo, excepto «Abadía», "Baños», «Colegiata» $\mathrm{y}$ «Hospital» que tienen dos y «Monasterio o Convento» con tres (Figura 7).

Algunos elementos como «Ermita» $\mathrm{y}$ «Monasterio o convento», aparecen de forma muy repetitiva en la mayoría de los 


\begin{tabular}{|c|c|c|c|c|}
\hline \multicolumn{2}{|c|}{ SIMBOLOGÍA CHEVALIER } & \multicolumn{2}{|c|}{ SIMBOLOGÍA AGE } & \multirow{2}{*}{$\begin{array}{c}\text { Coincide } \\
\end{array}$} \\
\hline $\begin{array}{l}\text { Torre o } \\
\text { Parroquia }\end{array}$ & clocher ou Paroisse & Ermita & $\phi$ & \\
\hline Castillo & Chattaue arec Avenues et Pare & Castillo & 1 & \\
\hline $\begin{array}{l}\text { Casa } \\
\text { Notable }\end{array}$ & - Maison notable & Casa fuerte & 1 & \\
\hline $\begin{array}{l}\text { Pueblo } 0 \\
\text { Aldea }\end{array}$ & Q Village ou Hameau & $\begin{array}{l}\text { Alquería/ } \\
\text { Aldea } \\
\end{array}$ & 0 & $\bullet$ \\
\hline Capilla & ¿ Chapelle & Capilla & t & $\bullet$ \\
\hline $\begin{array}{l}\text { Cortijo o } \\
\text { Finca } \\
\text { Aislada }\end{array}$ & $\Delta$ Ferme saule & $\begin{array}{l}\text { Cortijo o } \\
\text { caserios }\end{array}$ & 0 & • \\
\hline $\begin{array}{l}\text { Molino de } \\
\text { Viento }\end{array}$ & I Moulin-á-vent & Molino & $\not x$ & $\bullet$ \\
\hline $\begin{array}{l}\text { Molino de } \\
\text { Agua }\end{array}$ & * Moulin-á-eau & $\begin{array}{l}\begin{array}{l}\text { Azaña } \\
\text { (molino de } \\
\text { agua) }\end{array} \\
\end{array}$ & & \\
\hline Abadía & ๔ Abbaye & Abadia & $\oint$ & • \\
\hline Monasterio & $\delta$ Priauré & Monasterio & $\delta$ & • \\
\hline Convento & 5 Cawent & Convento & \lceil & \\
\hline Ruinas & $==$ Ruines & $\begin{array}{l}\text { Pueblo en } \\
\text { ruinas }\end{array}$ & 4 & \\
\hline $\begin{array}{l}\text { Lugar de } \\
\text { Combate }\end{array}$ & $\propto$ Lieu de Combat & - & - & \\
\hline $\begin{array}{l}\text { Horca de } \\
\text { ajusticiar }\end{array}$ & i Gibet ou Chustice & - & - & \\
\hline Puente & $\sqrt{\text { Pont }}$ & Puente & $\Rightarrow$ & $\bullet$ \\
\hline Río & $\approx$ Riviere & Rio & $\infty$ & $\bullet$ \\
\hline Isla & Isle & Isla & $\sqrt{n}$ & $\bullet$ \\
\hline Barca & $\widetilde{\widetilde{B a c}}$ & Barca & = & \\
\hline Arroyo & Ruisseau & Arroyo & & $\bullet$ \\
\hline Estanque & $*$ Etang & Laguna & & $\bullet$ \\
\hline Camino & ...-Chemin & Camino & $\because \ldots$ & $\bullet$ \\
\hline Bosque & 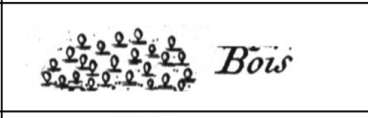 & Bosque & 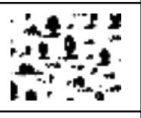 & $\bullet$ \\
\hline Brezo & Bruiére & $\begin{array}{l}\text { Arbolado } \\
\text { espaciado }\end{array}$ & 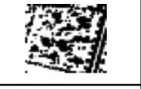 & $\bullet$ \\
\hline Viña & 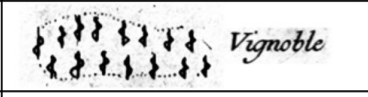 & Huertas & 69 & $\bullet$ \\
\hline $\begin{array}{l}\text { Altura } 0 \\
\text { ladera }\end{array}$ & Hauteur ou côteau & $\begin{array}{l}\text { Altura o } \\
\text { ladera }\end{array}$ & $\mathbb{W}_{\mathrm{E}}$ & $\bullet$ \\
\hline $\begin{array}{l}\text { Villa } \\
\text { amurallada } \\
\text { con } \\
\text { suburbios } \\
\end{array}$ & fot ville muré & Plaza fuerte & & $\bullet$ \\
\hline
\end{tabular}

Figura 4. Símbolos coincidentes en la representación de la plantilla de Chevalier con las señales del $A G E$ de Tomás López. 


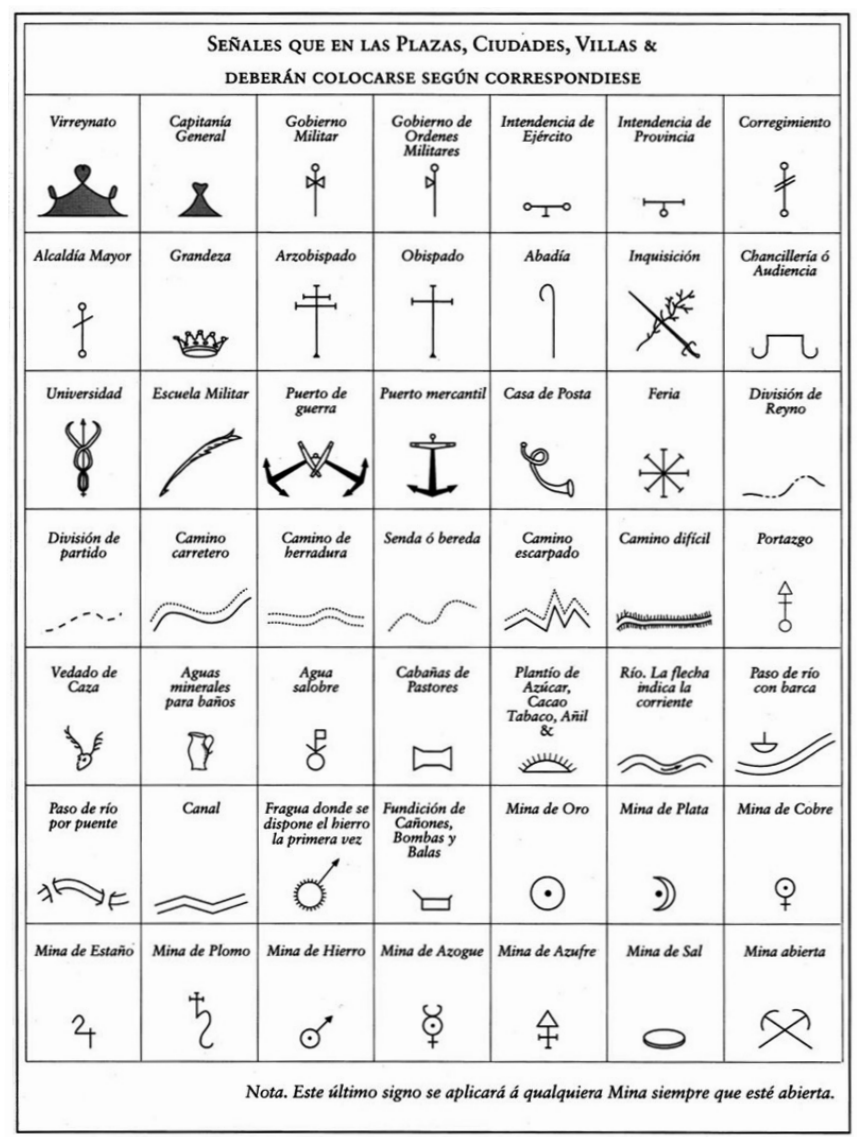

Figura 5. Señales cartográficas de las Ordenanzas del Real Cuerpo de Ingenieros del Ejército de 1803 (González Tascón, 1992).

mapas, lo que da idea de la gran cantidad de edificios de este tipo por todo el país. Se dan casos, como en el mapa del Obispado y Reino de Murcia (Hoja 69 del $A G E$ ), en que Tomás López agrupa con un mismo símbolo los «Monasterios» y las «Ermitas», lo que podría deberse a que no identificara exactamente el elemento del que se trataba, o que se asocien ambas funciones en un mismo edificio. Por otro lado, hay casos como el del mapa del Reino de Aragón (Hojas 70 a 73), donde

\begin{tabular}{|c|c|c|c|}
\hline $\begin{array}{c}\text { Señales } \\
\text { Ordenanzas } \\
1803\end{array}$ & \multicolumn{2}{|c|}{ Señales del AGE de Tomás López } & $\begin{array}{c}\text { Señales } \\
\text { Ordenanzas } \\
1803\end{array}$ \\
\hline $\begin{array}{l}\text { Camino de } \\
\text { herradura }\end{array}$ & \multicolumn{2}{|c|}{ Irozos de las Vias Militaras } & \\
\hline 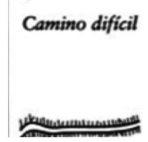 & \multicolumn{2}{|c|}{$\approx$ Caminos abiertos en Sierra Nevada } & $\begin{array}{c}\text { Camino } \\
\text { escarpado }\end{array}$ \\
\hline $\begin{array}{c}\text { Division de } \\
\text { partido }\end{array}$ & $\begin{array}{l}\text { Divide los } \\
\text { partidos. }\end{array}$ & $\approx i=P$ Puente & $\begin{array}{l}\begin{array}{c}\text { Paso de rio } \\
\text { por puente }\end{array} \\
\lambda \overbrace{F}\end{array}$ \\
\hline Arzobispado & †Arzobispacator & Fobispaido & $\begin{array}{l}\text { Obispado } \\
\text { † }\end{array}$ \\
\hline
\end{tabular}

Figura 6. Señales análogas en la representación de las Ordenanzas del Real Cuerpo de Ingenieros del Ejército de 1803 con las del AGE de Tomás López.

se reseña específicamente que «Ermita» se refiere a «Capilla/ Ermita de Nuestra Señora».

Otro elemento que aparece con frecuencia es «Venta», lo que da idea de la importancia que suponía conocer su ubicación en los caminos, teniendo en cuenta que los medios de transporte de la época eran de tracción animal. En cuanto al elemento «Universidad», es interesante comprobar que las universidades se localizaban, especialmente, en la parte norte y levante del país.

Junto a los símbolos anteriores, Tomás López también utiliza unos convencionalismos gráficos más visuales como en los casos de «Ermita» o "Iglesia», en donde además de representarlos con el símbolo de la Figura 7, los dibuja en alzado o perfil abatido, según una acreditada tradición cartográfica. Otro caso singular que es el de «Almacén» que no tiene símbolo definido en la Figura 7, pero que hay algún ejemplo también en perfil abatido (Figura 8).

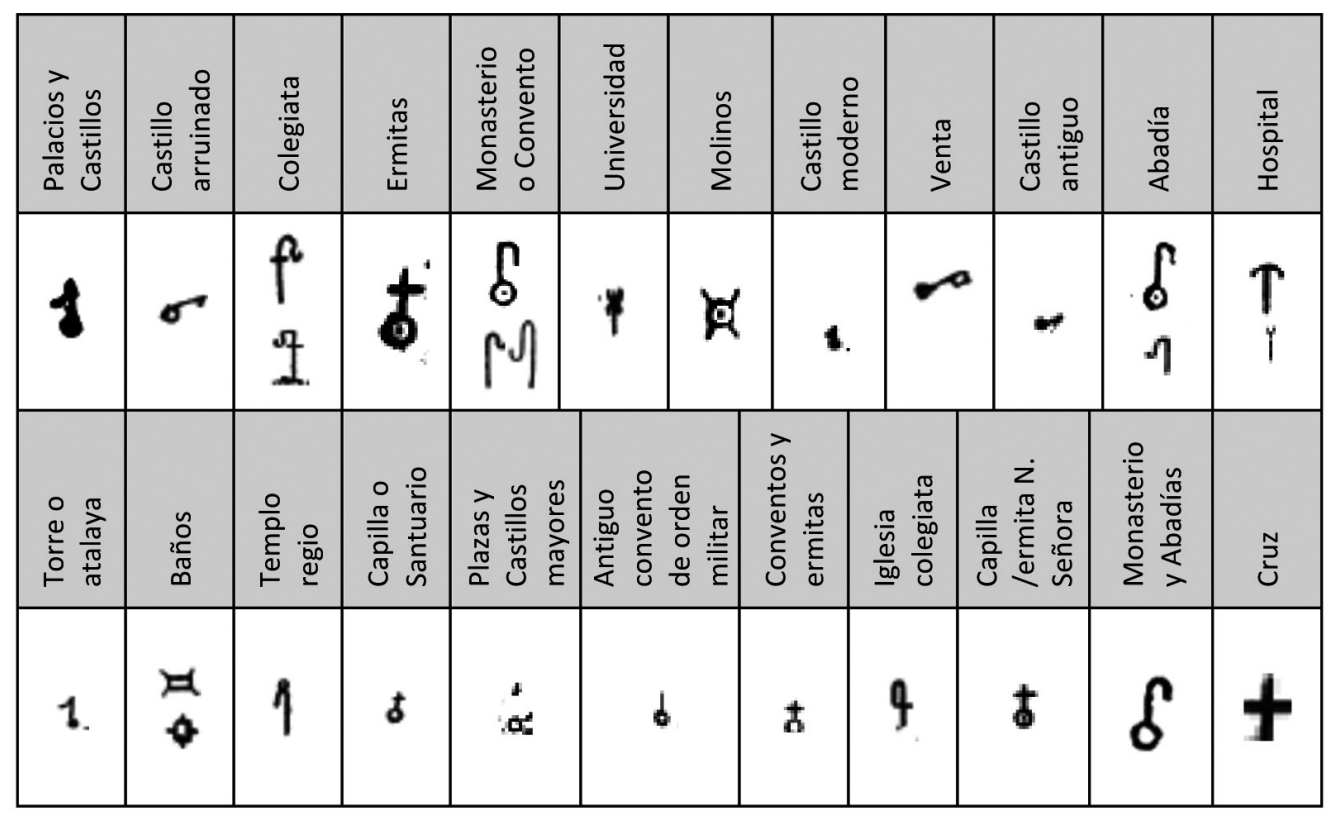

Figura 7. Símbolos de los elementos arquitectónicos. 


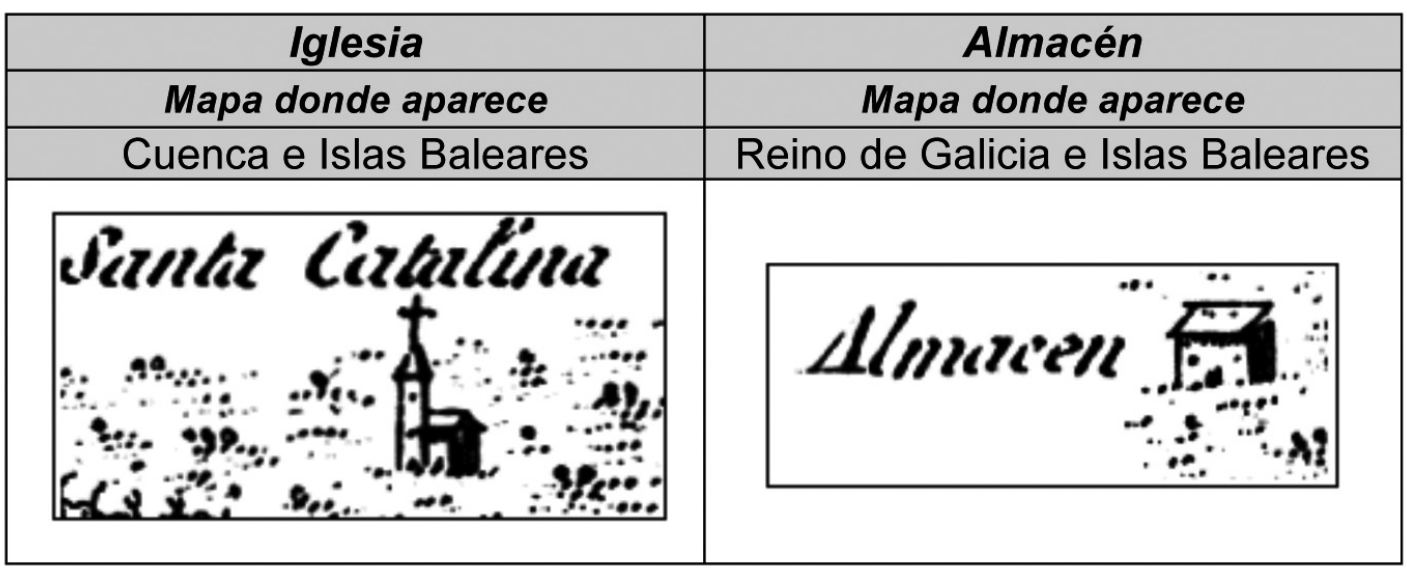

Figura 8. Tipologías en perfil abatido de elementos arquitectónicos.

\subsection{Modelo tipológico elementos urbanos}

Este modelo, con el de Administración civil y militar, es el que más elementos gráficos presenta con un total de treinta y ocho símbolos, lo cual confirma que es el primer Atlas que representa la gran variedad de núcleos de población del Reino de España y, en su caso, de Portugal. Sus elementos son los siguientes: Cortijos, caseríos, granjas, dehesas, Lugar o aldea, Villa Grande, Villa Mediana, Villa, Despoblado, pueblo en ruinas, Lugar grande, Barrio o vecindario, Lugares y Granjas eximidas, Villa menor, Villa regular, Nueva Población, Villas y lugares con jurisdicción, Lugar y Parroquia, Parroquia, Lugar sin Parroquia, Cabeza de Concejo, Villa cabeza de partido, Villas y lugares de Valdavia, Villas del Partido, Término sin casa, Villas sueltas, Lugar menor, Lugar, Aldea, Villa con asiento en cortes, Quadra, Término, Villas que celebran Juntas Generales, Villas con asiento en Juntas, Pueblos con asiento en Juntas agregados, Behetría, Plaza, Anteiglesias con voto para Juntas, Anteiglesias adheridas para Juntas y Alquería.

Como se observa en la anterior relación, Tomás López estableció una definición de los distintos asentamientos urbanos según su tamaño. Así habla, por ejemplo, de «Villa grande», «Villa menor», «Villa regular», «Cortijo» y «Despoblado». También se distinguen en las leyendas otros elementos menores, desde el punto de vista poblacional, como es el caso de «Barrio» o «Vecindario». La clasificación de los elementos gráficos según su tamaño, es la que más se repite en las leyendas del $A G E$. Aunque hay treinta y ocho elementos, sin embargo en los mapas del $A G E$, aparecen cuarenta y tres símbolos gráficos, ya que «Lugar grande», «Villas, Lugares y Granjas eximidas» $\mathrm{y}$ «Villa con asiento en cortes» se representan indistintamente con dos símbolos y «Barrio o vecindario» con tres (Figura 9).

Asimismo se han caracterizado los elementos urbanos según su jerarquía en la administración religiosa, como «Lugar y parroquia» o «Lugar sin parroquia». También los distintos asentamientos se representan por su relevancia en la administración civil, como las «Villas del partido», «Cabeza de partido», «Cabeza de concejo», «Villas y lugares con jurisdicción». Un caso particular corresponde al País Vasco, donde por tener fueros propios, en la Provincia de Guipúzcoa se representan por un lado las «Villas con asiento en Juntas» y, por otro, los pueblos con «Asiento en Juntas agregados». Lo mismo pasa en el Señorío de Vizcaya, donde se representan las «Anteiglesias con voto para Juntas» y las «Anteiglesias adheridas para Juntas».

En general, la escala de los mapas del $A G E$, no permite representar los planos de los núcleos urbanos: lugares, pueblos, villas, etc., más que con los símbolos normalizados de la Figura 9. En cuanto a las ciudades, la mayoría aparecen con esos símbolos, aunque en otras, se dibujan en planta con una forma esquemática más elaborada como Valencia y Lisboa (Figura 10). Caso único en el $A G E$, es el plano de Oviedo que ocupa toda la Hoja $n .{ }^{\circ} 33$. Su escala, a partir de la escala gráfica ( 180 varas $=65 \mathrm{~mm}$ ), es E: 1/2314,8 (considerando que 1 vara castellana equivale a $835,9 \mathrm{~mm}$ ). Es un plano detallado de la ciudad con sus manzanas, calles, plazas, edificios singulares y su entorno a extramuros. La edificación aparece con un rayado a $45^{\circ}$ donde se destacan los edificios singulares con letras mayúsculas. Las calles y plazas se numeran desde el centro de la ciudad hacia el extrarradio con su nombre correspondiente. $\mathrm{El}$ arbolado y la vegetación se representan en perfil abatido (Figura 10).

\subsection{Modelo tipológico obras de ingeniería civil}

Son dieciocho sus elementos representados: Caminos, Caminos reales, Puentes, Puente arruinado, Canal proyectado, Canal concluido, Curso del canal, Puerto de Mar, Barcas, Caminos principales, Caminos menores, Caminos y senderos, Otro camino, Trozos del camino de la Plata, Caminos abiertos en S. Nevada, Camino real nuevo tirado a cordón, Camino regular, Camino M. Marquesado, Camino Nuevo, Barcas en servicio desde 1704 y Puente de Barcas.

Todos ellos parecen en el $A G E$, pero no de forma sistemática en cada uno de sus mapas. Los símbolos que más se prodigan son «Caminos», «Puentes» y "Barcas», aunque no estén en todos los mapas. Estos tres símbolos son la base de la ingeniería civil en la obra analizada y debían de ser puntos críticos de las vías de comunicaciones de la época. Hay que reseñar también que en algún mapa concreto, estos símbolos se detallan con alguna particularidad como es el caso de «Caminos abiertos en S. Nevada» o «Barcas en servicio desde 1704 para el paso de las tropas de Felipe V» (Figura 11).

En la Figura 11 aparecen nueve símbolos para representar los caminos. A continuación ponemos ejemplos de las cinco tipologías mejor definidas en el $A G E$ según la importancia y anchura del camino. Éstas tipologías se dan en todo el $A G E$ me- 


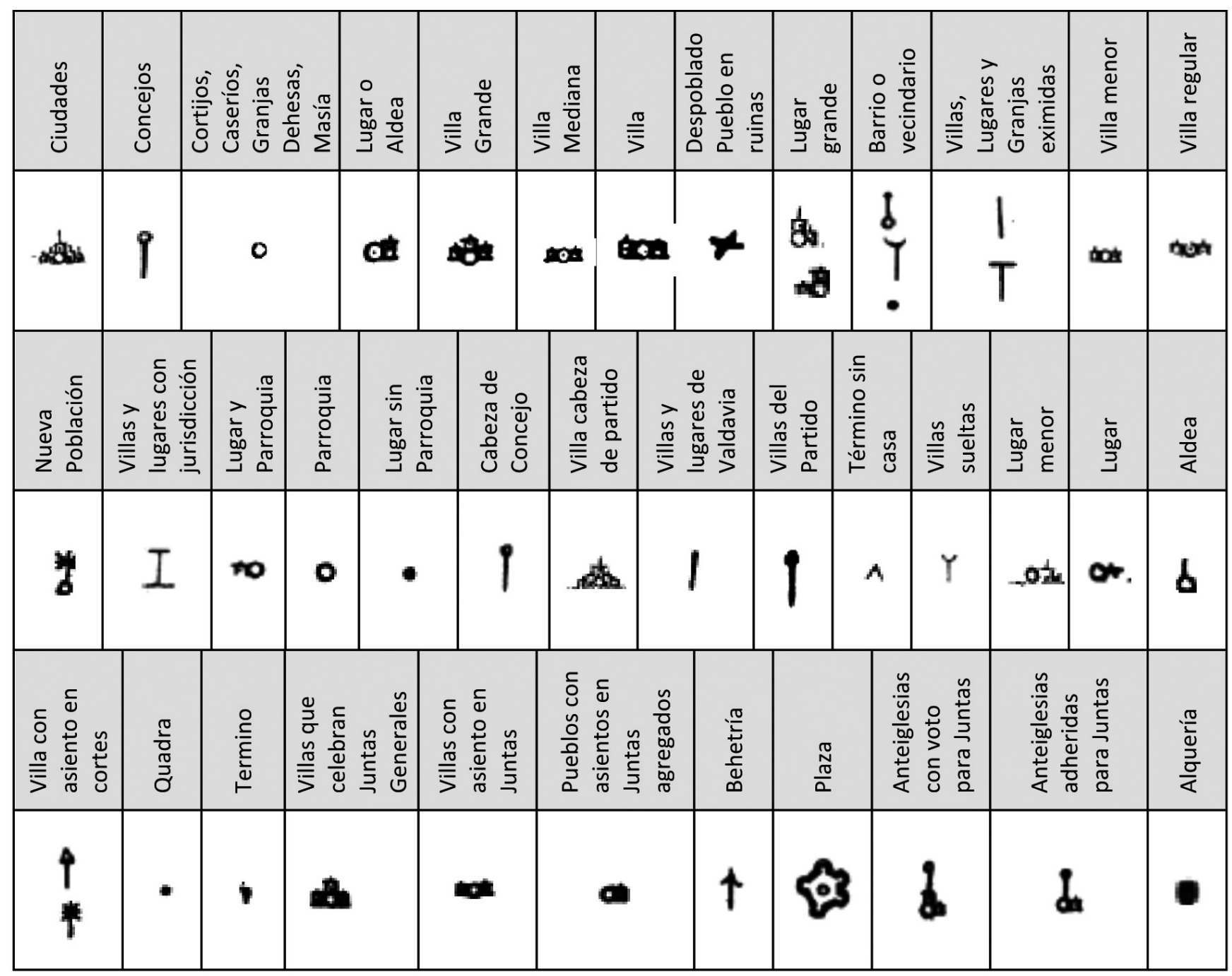

Figura 9. Símbolos de los elementos urbanos.

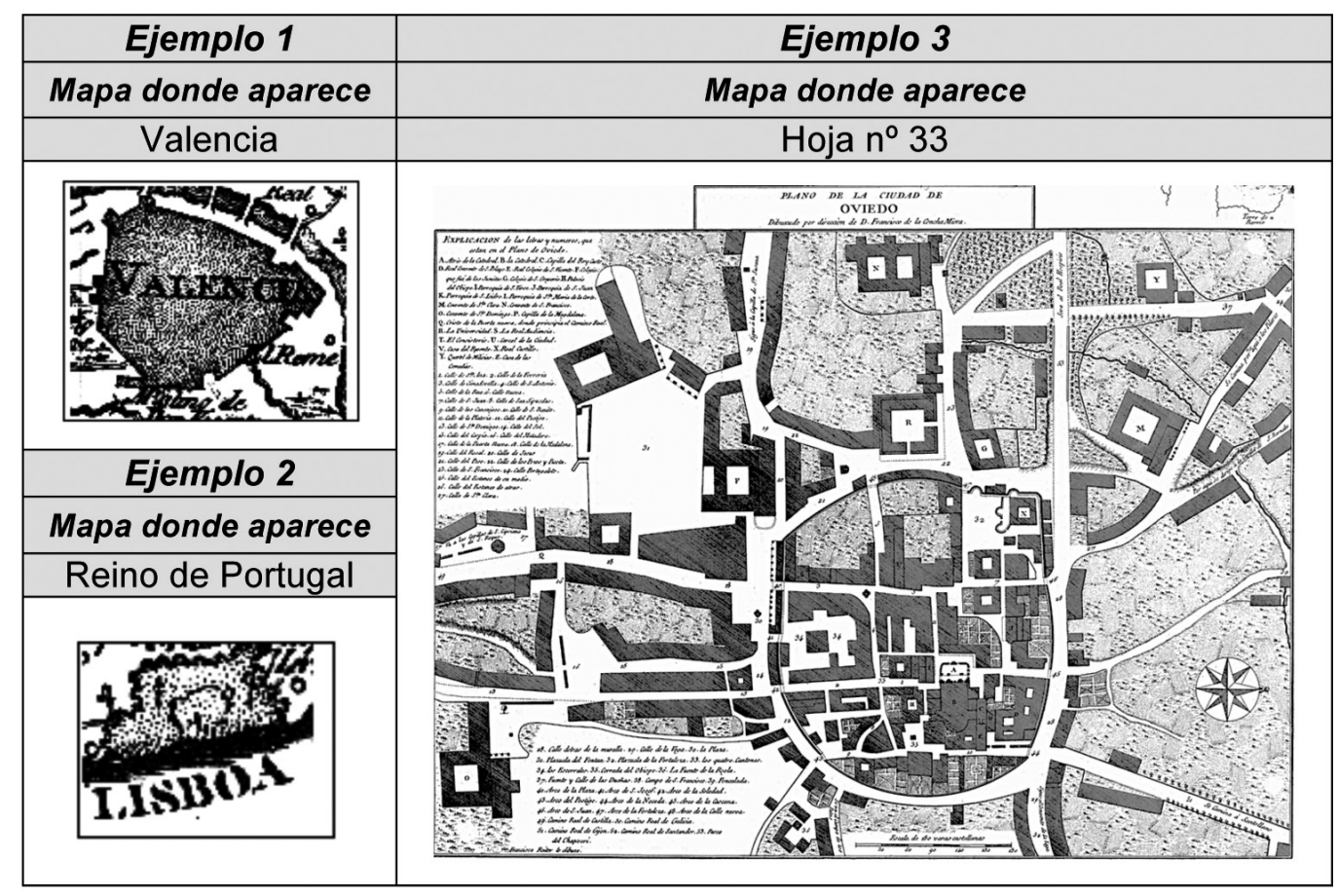

Figura 10. Ejemplos de tipologías para representar los núcleos urbanos. 


\begin{tabular}{|c|c|c|c|c|c|c|c|c|}
\hline 号 & 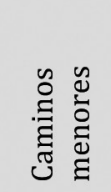 & 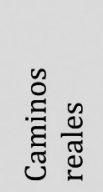 & 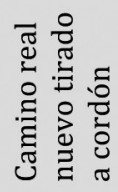 & 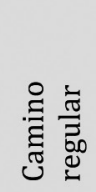 & 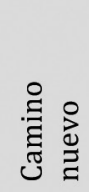 & 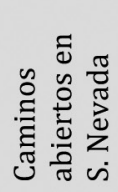 & 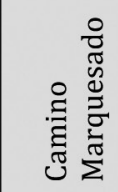 & 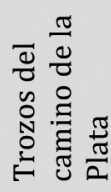 \\
\hline$\sim$ & $\longrightarrow$ & $\sim$ & $\infty$ & & $\sim$ & $\infty$ & |- & נرخ \\
\hline 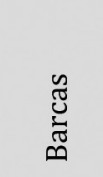 & 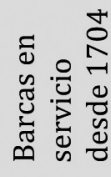 & 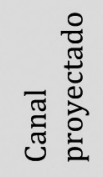 & 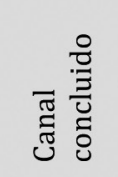 & 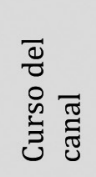 & 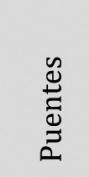 & 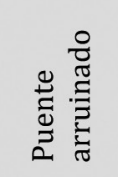 & 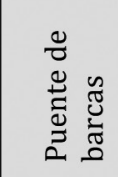 & $\begin{array}{l}\stackrel{0}{0} \\
0 \\
0 \\
\frac{0}{2} \\
\Sigma \\
\Sigma \\
\Sigma\end{array}$ \\
\hline 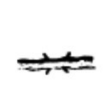 & I & $\infty$ & $\ldots$ & $\approx$ & $\Rightarrow$ & $\Longrightarrow$ & x & \\
\hline
\end{tabular}

Figura 11. Símbolos de las obras de ingeniería civil.

nos es el mapa General de las Islas Baleares y Pitiusas, donde no se representa camino alguno. Las cinco tipologías son:

- Tipología 1. Con línea simple discontinua.

- Tipología 2. Con línea simple continua.

- Tipología 3. Con línea doble discontinua.

- Tipología 4. Con línea doble continua.

- Tipología 5. Caminos singulares.

Esta última tipología aparece en la Hoja 1 del mapa de Madrid, en el camino de Aranjuez, como una doble línea continua con un rayado perpendicular a su dirección que simula cunetas y desmontes o terraplenes. También, en el trazado urbano de Aranjuez, los caminos se representan por una doble línea de puntos, donde se detallan, incluso, las glorietas del núcleo urbano. Igualmente nos encontramos con los caminos de Sierra Nevada, representados como dobles líneas poligonales abiertas a cuyos lados se dibujan sendas líneas de puntos simulando los desmontes o terraplenes. Estos caminos parecen dibujar en su trazado una serie de líneas estructurales del terreno.

Las tipologías 2, 3 y 4 son las que se dan de forma mayoritaria en los diferentes mapas, mientras que las 1 y 5 son excepcionales. Elegimos a continuación un ejemplo de cada una de ellas (Figura 12).

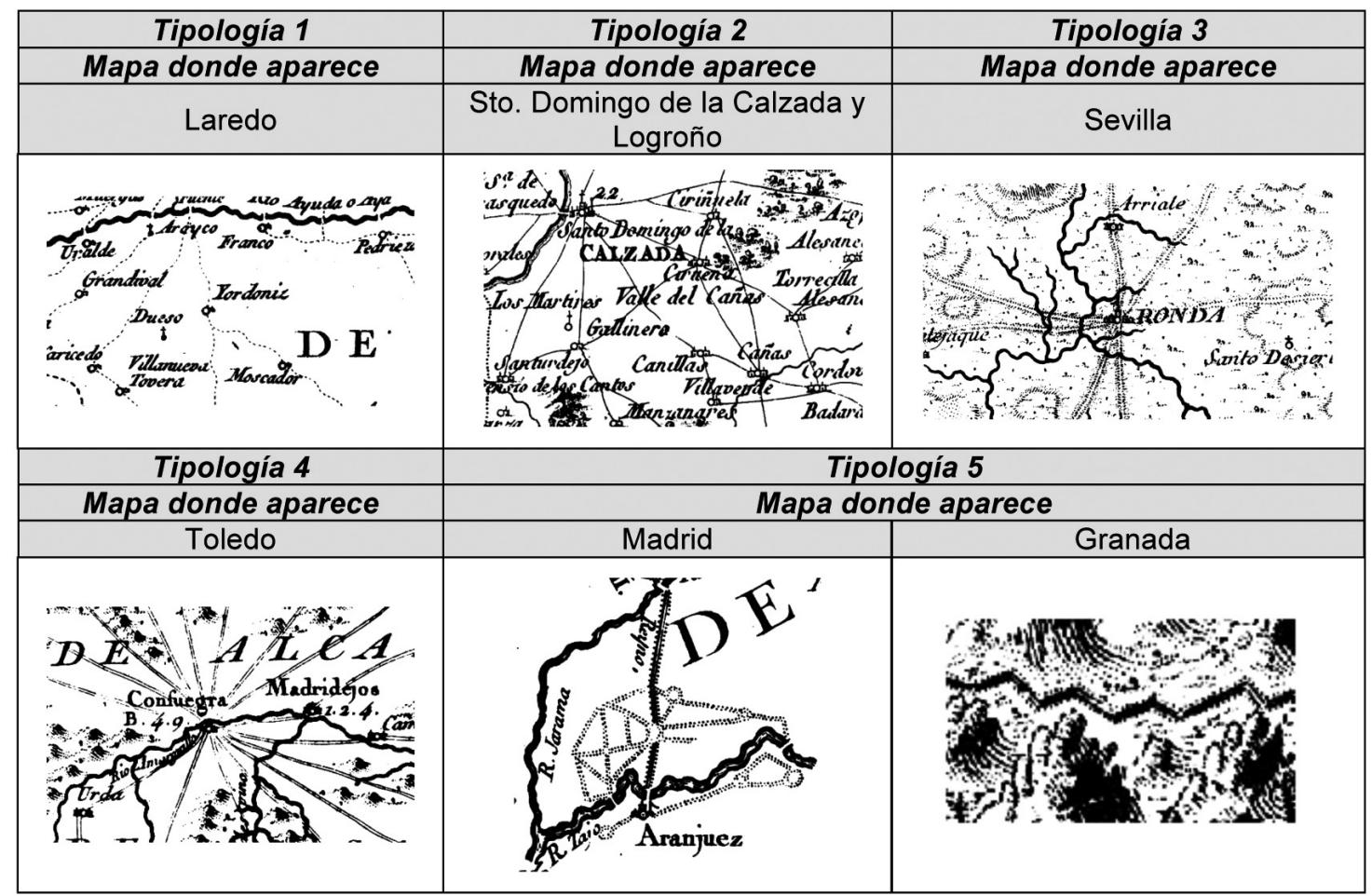

Figura 12. Ejemplos de tipologías para representar los caminos. 
Como ejemplos de la ubicación de algunos símbolos de los mapas en un contexto más amplio que el de su propia representación gráfica, tenemos los «Puentes», que se dibujan en planta con dos líneas paralelas abiertas en los extremos; los «Canales en uso", que se dibujan con dos líneas paralelas sobre las que se adosan sendas líneas de puntos, y los «Canales proyectados», que se representan con esas dos líneas paralelas rayadas con trazos perpendiculares a ellas. Otro caso es el «Puente de barcas», representado con un símbolo parecido al del «Puente de obra», pero cerrado en los extremos en su conexión con ambas riberas, de tal forma que aparece como un rectángulo que atraviesa el río del que salen segmentos a $45^{\circ}$ en sus vértices. El «Puente de barcas» es, junto con los «Puentes», el más representado en el $A G E$. En cuanto a los «Puertos», se representan con un ancla situada en la zona donde está construido. En el caso de puertos naturales, se dibuja la costa y se pone la leyenda de «Puerto» con su nombre. Es el caso de Puerto Pi
(Hoja 82) y el de la Isla de Ibiza (Hoja 83). Un caso único es el del acueducto de Segovia que aparece junto a la cartela de esa provincia que corresponde a la Hoja 19 del AGE. Todos estos casos aparecen en la Figura 13.

\subsection{Modelo tipológico obras de ingeniería militar}

Este modelo tan solo tiene nueve elementos gráficos diferentes y aparece en pocas provincias: Fortificación, fuerte, Lugar fuerte o Castillo, Trozos de vías militares, Plaza de Armas, Lugar fuerte, Sitio fuerte, Castillo o casa fuerte, Palacio o casa fuerte y Plaza fuerte. Los símbolos son muy similares con la única excepción de «Torres de vigilancia costera», que aparece representado en alzado solamente en el mapa de las Islas Baleares. En cuanto al símbolo de un banderín que se apoya sobre un pequeño círculo con su interior en blanco, se repite en «Castillo», «Palacio o casa fuerte» $\mathrm{y}$ «Torre» (Figura 14).

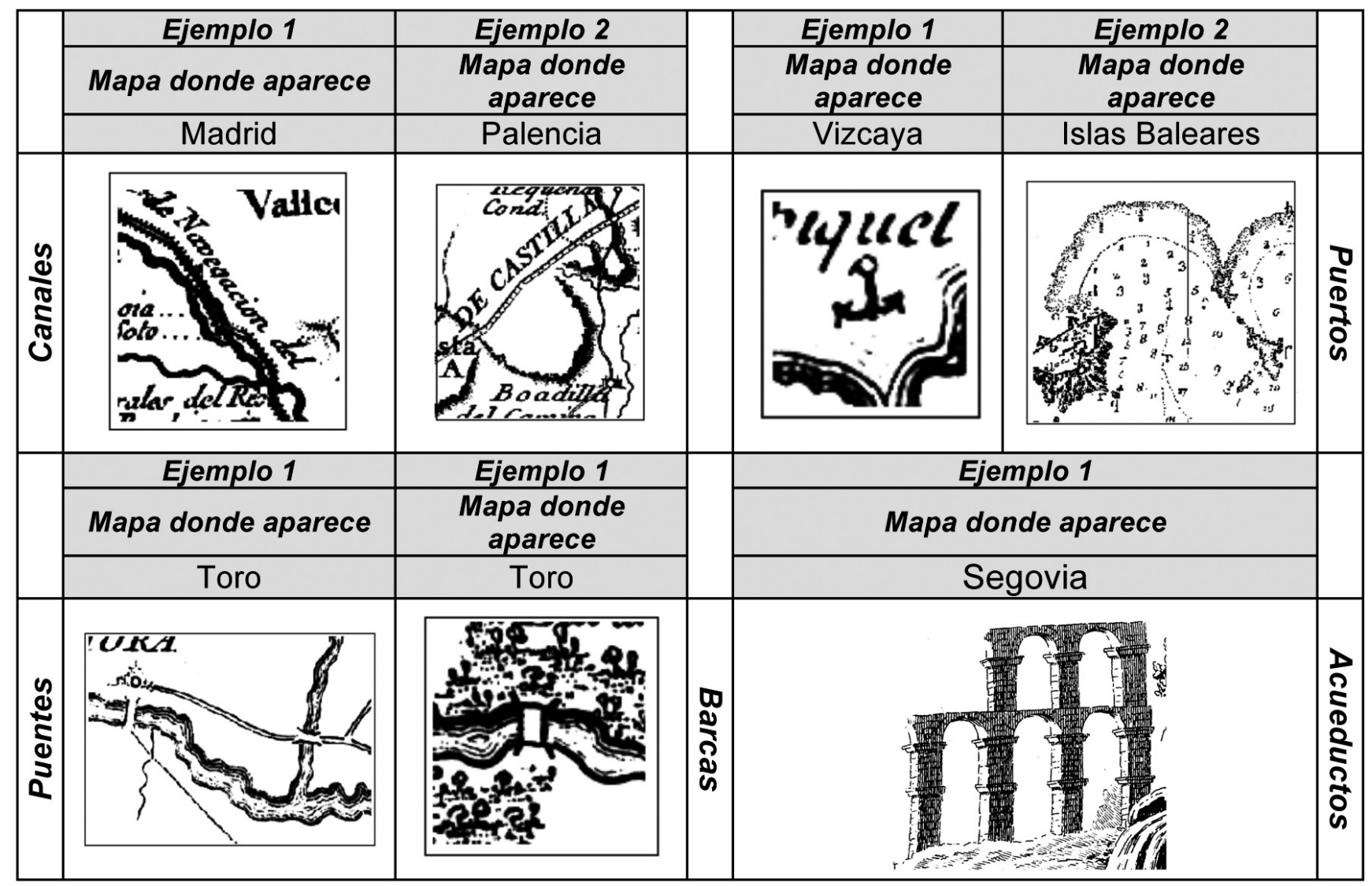

Figura 13. Ejemplos de tipologías para representar acueductos, barcas, canales y puentes.

\begin{tabular}{|c|c|c|c|c|c|c|c|c|}
\hline 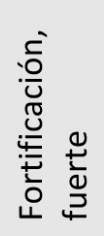 & 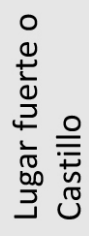 & 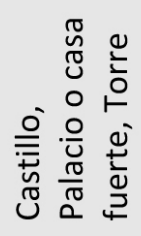 & 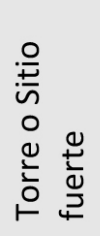 & 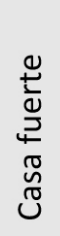 & $\begin{array}{l}\underset{0}{\frac{\pi}{2}} \\
\stackrel{0}{2} \\
\frac{\pi}{N} \\
\frac{\pi}{\alpha} \\
\frac{\pi}{\alpha}\end{array}$ & 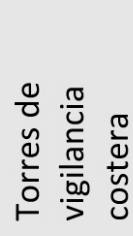 & 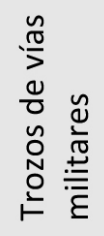 & $\begin{array}{l}\frac{\pi}{0} \\
\mathbb{N} \\
\mathbb{N} \\
\frac{\pi}{2} \\
\end{array}$ \\
\hline & & & & & & & & $x$ \\
\hline
\end{tabular}

Figura 14. Símbolos de las obras de ingeniería militar. 
Un caso aparte es el de las ciudades fortaleza con escaso caserío en su interior que se dibujan en planta y con muy poco detalle como, por ejemplo, la ciudad de Ibiza (Figura 15), a diferencia de cómo se representan otra ciudades amuralladas muy consolidadas como Valencia o Lisboa, con su interior sombreado mediante un punteado (Figura 10). Volviendo al caso ya visto de ciudad de Oviedo, como único ejemplo de ciudad amurallada representada a una escala suficiente, la muralla se dibuja con doble línea negra/blanca, trazada una junto a otra, interrumpidas por los huecos de las puertas de entrada a la ciudad (Figura 15).

\section{CONCLUSIONES}

Para analizar la simbología con la que Tomás López representa la arquitectura, el urbanismo y las obras públicas civiles y militares en el $A G E$, se ha diseñado un catálogo con los recursos gráficos que utilizó y que constituyen los parámetros de referencia del análisis. La gran variedad de convenciones gráficas caracterizadas, permiten transmitir información fidedigna del significado de lo representado y de su forma. Se han encontrado un total de 88 símbolos o señales diferentes: 23 para elementos arquitectónicos, 38 para elementos urbanos, 18 correspondientes a las obras de ingeniería civil y 9 para las obras de ingeniería militar. Junto a estos símbolos, aparecen otros 18 convencionalismos gráficos más visuales, con los que busca un mayor parecido con la realidad. Tenemos así un total de 106 recursos gráficos diferentes que dan idea de la minuciosidad y riqueza de la información de los mapas del $A G E$.

Se ha encontrado equivalencia entre 18 símbolos del AGE con otros tantos símbolos cartográficos de Chevalier, maestro de D’Anville de quien Tomás López fue discípulo, lo que pone de manifiesto su formación francesa. Hay también un paralelismo entre 4 de las señales de Tomás López con sus correspondientes de las Ordenanzas del Real Cuerpo de Ingenieros del Ejército de 1803.

Aunque la simbología es común en la mayoría de los mapas del $A G E$, se da el caso en el que se utiliza un mismo símbolo para identificar elementos gráficos diferentes. Por ejemplo, en el modelo tipológico Obras de ingeniería militar, aparece el mismo símbolo 1 referido a «Lugar fuerte o castillo», «Castillo, palacio o casa fuerte, Torre» (Figura 14).

También hay elementos gráficos con igual denominación y distinta simbología, dependiendo del mapa en el que aparez-

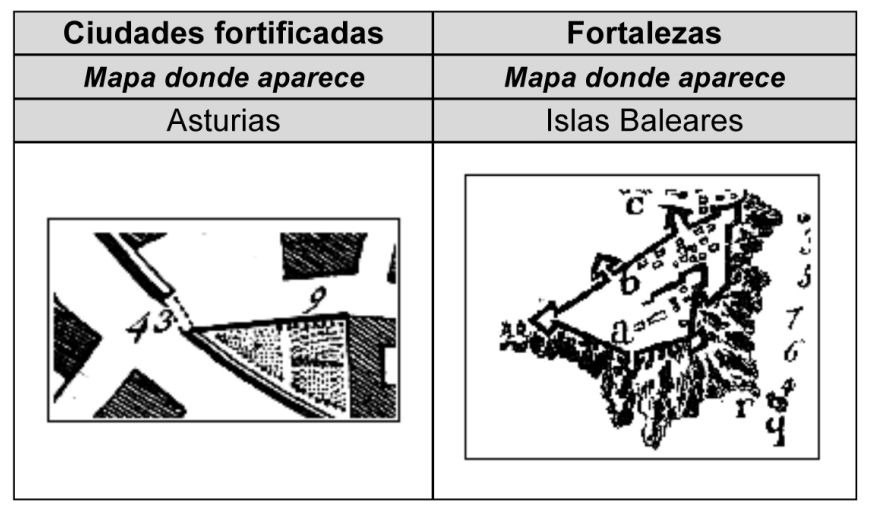

Figura 15. Ejemplos de tipologías para obras de ingeniería militar. can, aunque sus representaciones gráficas sean bastante similares. Como ejemplo tenemos el «Monasterio o convento», del modelo tipológico Elementos arquitectónicos, que según se encuentre en uno u otro mapa, se representa con los símbolos 5, , o f . Otro caso es el del elemento «Colegiata» que dependiendo de los mapas, aparece con los símbolos f o £. También, el elemento "Abadía", se representa con la simbología § o л según el mapa del que se trate (Figura 7). Todos estos ejemplos, aunque pertenecen a Elementos arquitectónicos, se dan también en otros modelos tipológicos $\operatorname{del} A G E$

Otro caso anómalo es el de elementos diferentes que en unos mapas aparecen con el mismo símbolo y en otros con otro distinto. De nuevo tenemos el elemento «Abadía», del modelo tipológico Elementos arquitectónicos, que en unos mapas se representa con el símbolo §, el mismo con el que en otros mapas se representa "Monasterio». En otros mapas «Abadía» se representa con el símbolo ^ y Monasterio con la grafía 0 (Figura 7).

Estos casos muestran que Tomás López no dio demasiada importancia a la reglamentación de los símbolos, siempre que su representación gráfica tuviera cierto grado de normalización y, que en el caso de variar su grafía, los distintos símbolos conservaran cierta analogía.

Cabe decir a favor de Tomás López que, para cada mapa, los distintos símbolos o señales, tienen una única forma de representación gráfica, que es distinta de la atribuible a cualquiera de los otros elementos, lo que hace perfectamente identificable cada uno de ellos en un mapa concreto.

Otro punto a considerar es que hay mapas en los que un cierto elemento se representa de una manera singular y no como en el resto de mapas. Tal es el caso de «Barcas en servicio desde 1704 sobre el río Tajo, para el paso de las tropas de Felipe $V »$, perteneciente al modelo tipológico Obras de ingeniería civil, y se representa con la grafía I (Figura 11). En este caso, Tomás López ha querido diferenciar este elemento, que aparece en un mapa del Reino de Portugal (Lámina 93 del $A G E$ ), del símbolo que es el que habitualmente utiliza en el resto de los mapas provinciales para representar «Barcas». Esta particularidad puede deberse a que quiso reflejar la fecha de 1704, que corresponde a la campaña de Portugal (marzo-julio 1704), durante la Guerra de Sucesión a la Corona de España, en la que las tropas de Felipe V impidieron que los ingleses entraran en España.

\section{AGRADECIMIENTOS}

Este estudio fue financiado con el proyecto de Investigación HAR2009-12937 del Plan Nacional I+D+i: Análisis sistemático mediante tecnología GIS de la precisión planimétrica de los mapas del Atlas Geográfico de España de Tomas López de 1804.

Al CNIG (Centro Nacional de Información Geográfica), por las imágenes de todos los mapas del $A G E$ cedidas para esta investigación, de las que proceden cada uno de los símbolos utilizados por Tomás López. También por su edición facsímil del $A G E$ (ISBN: 84-9810-436-X) que tan amablemente nos facilitaron. 


\section{REFERENCIAS}

(1) Manzano Agugliaro, F., Martínez García, J., San Antonio Gomez, J. C. (2012). GIS analysis of the accuracy of Tomas Lopez's historical cartography in the canary islands (1742-1746). Scientific Research and Essays, 7(2): 199-210, doi: http://dx.doi.org/10.5897/SRE11.1271.

(2) Chías, P., Abad, T. (2012). El arte de describir el territorio: mapas y planos históricos en torno al puente de Alcántara (Cáceres, España). Informes de la Construcción, 64 (Extra): 121-134, doi: http://dx.doi.org/10.3989/ic.11.071.

(3) San Antonio Gómez, C., Velilla, C., Manzano Agugliaro, F. (2011). Tomas Lopez's geographic atlas of Spain in the Peninsular war: A methodology for determining errors. Survey Review, 43(319): 30-44, doi: http://dx.doi.org/10.1179/oo39 $62610 X 12747001420861$.

(4) López Gómez, A., Manso Porto, C. (2006). Cartografía del siglo xvin, Tomás López en la Real Academia de la Historia, p. 521. Madrid: Real Academia de la Historia.

(5) Sanz Hermida, J. M. (1992). Atlas Geográphico del Reyno de España e islas adyacentes. Salamanca: Europa Ediciones de Arte.

(6) Hernando, A. (2005). El Atlas Geográfico de España (1804) producido por Tomás López. Madrid: Instituto Geográfico Nacional. Centro Nacional de Información Geográfica.

(7) Clark, P. K., Jones, Y. (1974, 29 de abril). British military map making in the Peninsular war. En VII Conferencia Internacional de Cartografía ICA, p. 106. Madrid: International Cartographic Association.

(8) Núñez de las Cuevas, R. (2012). El poder de los mapas. Estudios Geográficos, 73(273): 587-588, doi: http://dx.doi. org/10.3989/estgeogr.201220.

(9) Manzano Agugliaro, F., Fernández Sánchez, J. S., San Antonio Gómez, C. (2013). Mapping the whole errors of Tomas Lopez's geographic atlas of Spain (1804). Journal of Maps, 9(4): 617-622, doi: http://dx.doi.org/10.1080/17445647.20 13.842188.

(10) Manzano Agugliaro, F., San Antonio Gómez, C., López, S., Montoya, F. G., Gil, C. (2013). Pareto-based evolutionary algorithms for the calculation of transformation parameters and accuracy assessment of historical maps. Computers and Geosciences, 57: 124-132, doi: http://dx.doi.org/10.1016/j.cageo.2013.04.010.

(11) Marcel, G. (1908). El geógrafo Tomás López y sus obras: ensayo de biografía y de cartografía. Boletín de la Real Academia de la Historia, LIII: 452-453. Madrid.

(12) Capel, H. (1982). Geografía y matemáticas en la España del siglo xviII. Barcelona: Oikos-Tau.

(13) Liter Mayayo, C., Sanchís Ballester, F. (1998). Tomás López y sus colaboradores. Madrid: Biblioteca Nacional.

(14) San Antonio Gómez, C. (2005). Castillos y ciudades fortificadas en la América Española: Planos del siglo xviIr. En Ribera, F. (ed.), Luci tra le rocce, vol. I, pp. 100-109. Firenze: Alinea Editrice s.r.l.

(15) San Antonio Gómez, C. (2006, 1-2 de junio). Metodología para el análisis gráfico de la cartografía histórica. En Congreso Internacional XVIII Ingegraf, pp. 81-95. Barcelona: Universidad Politécnica de Cataluña.

(16) Alinhac, G. (1965). Histoire de la Cartographie. París: Institut Géographique National.

(17) Chevalier M. (1707). Sur la Maniere de lever la carte d'un pays. Histoire de l'Academie Royal de Sciences, pp. 113-116. París.

(18) Manzano Agugliaro, F., Manzano Agugliaro, G., San Antonio Gómez, C. (2005, 1-3 de junio). El levantamiento topográfico y la cartografía en el siglo xvin: el método de la topografía eclesiástica. En Congreso Internacional XVII IngegrafXV ADM, pp. 284-294. Sevilla: Universidad de Sevilla.

(19) González Tascón, I. (1992). Ingeniería española en ultramar (siglos XVI-XIX), vol. I, p. 85. Madrid: Colegio de Ingenieros de Caminos, Canales y Puertos.

(20) Paladini Cuadrado, Á. (1989). Cartografia militar española de Indias. Militaria, Revista de Cultura Militar, 1: 57-89. Madrid: Ed. Univ. Complutense. 